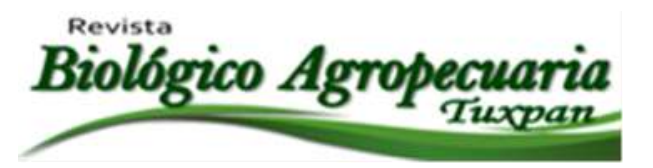

\title{
Análisis descriptivo de las MYPyMES agroindustriales de la región de Delicias, Chihuahua
}

Descriptive analysis of agro-industrial MYPyMES from the Delicias region, Chihuahua.

\author{
Magaña Magaña José Eduardo ${ }^{\bowtie 1}$, Chacón Márquez Aracely ${ }^{1}$, Villarreal Ramírez Víctor Hugo ${ }^{1}$, \\ Hermosillo Nieto José Javier ${ }^{1}$, Ortega Montes Fabiola Iveth ${ }^{1}$ \\ ${ }^{1}$ Facultad de Ciencias Agrícolas y Forestales de la Universidad Autónoma de Chihuahua \\ ${ }^{\otimes}$ Autor para correspondencia: emagana@ uach.mx
}

Recibido: $18 / 04 / 2020$

Aceptado: $24 / 05 / 2020$

\section{RESUMEN}

Esta investigación se realizó en el municipio de Delicias, Chihuahua en el año 2019. Se determinó analizar las capacidades productivas, emprendedoras y comerciales de las MIPyMES agroindustriales de producción y comercialización. Se utilizó la técnica de encuesta con un cuestionario de 33 preguntas con 188 variables, aplicados a 35 empresas. Los principales resultados que arrojo este estudio fueron que $36 \%$ son microempresas, $44 \%$ pequeñas empresas, $16 \%$ de medianas empresas y una gran empresa que representa el $4 \%$. Con base a la distribución de la planta, existe una participación mayor del $65.71 \%$ de trabajadores en el departamento de producción. El 52\% de las empresas no tienen una planeación estratégica como tal y tienen objetivos y metas de producción y ventas de acuerdo a sus compromisos y no como un documento de guía estratégica que implica la producción. En tanto el 12\% que pertenecen a las microempresas, manifestaron que no tiene nada de planeación estratégica. Entre las principales fortalezas se encontró que las empresas son dirigidas por personas con capacidad emprendedora y potencial para transformar de mejor forma, estrategias que les permita alcanzar un futuro deseable para la organización. Esto, hace que utilice sus recursos de la mejor forma para alcanzar sus objetivos, tales como recurso humano, equipo e infraestructura, ventas y canales de distribución. Sin embargo, se tiene que trabajar más en sus debilidades tales como capacitación, planeación estratégica e integración a las diferentes cámaras y organizaciones.

Palabras clave: Competitividad, capacitación, planeación, pequeñas empresas, sustentabilidad.

\begin{abstract}
This research was carried out in the municipality of Delicias, Chihuahua in 2019, it was determined to analyze the entrepreneuria productive capacites and needs. The business were selected from agribusiness industry particularly to those in production and comercialization. The technique wich was used were to apply questionare to 25 companies. The main results of this study was that $36 \%$ were micro-enterprises, $44 \%$ small bussinesses, $16 \%$ of medium - sized companies and a large company representing $4 \%$. Besides, the distribution plant gave us that a greater participation were $65.71 \%$ of worbers in the production department, $52 \%$ of companies did not have a strategic planning as such and had not production and sales objetives and goals according to their commitments therefor did not had a strategic guide document that involves production while $12 \%$ belong to micro - enterprises stated that they have no strategic. The main strengths were that companies were led by people with entrepreneurial capacity and potencial to better transform strategies that allow them to achieve a desirable future for the
\end{abstract}


organization. This means that they have to use their resources in the best way to achieve objectives such as human resources, equipment and infrastructure, sales and distribution channels. However, they have to work more on the weaknnesses like training, strategic planning, integration and organization.

Keywords: Competitiveness, trainnig, planning, small companies, sustainability.

\section{INTRODUCCIÓN}

El panorama para las pequeñas y medianas empresas (Pymes) de México es poco halagador de acuerdo a Arana (2018), en donde se manifiesta poco crecimiento, bajo aumento en la escala de producción, poco cambio en el tamaño de las empresas y difícil acceso al sistema crediticio. Bajo estas condiciones y aunado a cambios de política económica se hace necesario analizar la situación actual de cómo están realizando su gestión empresarial y proponer alternativas de cambio para aprovechar las oportunidades y reforzar sus fortalezas.

En el año 2018, el Instituto Nacional de Estadística y Geografía (INEGI), informo que en México están operando 4.2 millones de unidades económicas. De los cuales, 4.19 millones $(99.8 \%)$ están clasificadas como Pymes. Las Pymes son las responsables de generar el $78 \%$ del total de los empleos formales en este país y contribuyen con el $42 \%$ del Producto Interno Bruto (PIB). En Chihuahua existen 122, 092 empresas, siendo el 99\% de Pymes. Que aportan el 50.4\% del PIB estatal y generan el $68 \%$ del empleo del estado INEGI (2018).

En el estado de Chihuahua, las Micro, Pequeñas y Medianas Empresas (MIPyMES) desempeñan un papel estratégico en la generación de empleo después de la maquila, importante son las agendas municipales que demandan información de la estructura empresarial, comercial y de servicios que ayudan a medir indicadores de desarrollo económico y a fortalecer sus capacidades institucionales que mejoran el desempeño de sus funciones que las constituyen, las cuales contribuirán a mejorar la calidad de vida de la población.

La disminución del apoyo gubernamental federal, entre otros, plantea la necesidad de encontrar nuevas soluciones de mejora en el capital humano, capital financiero, capital de equipo e infraestructura y capital relacional para incrementar niveles de productividad, competitividad y continuidad en el mercado de las MIPyMES. A pesar de las prohibiciones que las MIPyMES pueden tener en la economía, han forjado que la productividad aumente, valor agregado, consolidación del mercado interno, acrecimiento de importaciones, entre otros, por lo que se han suscitado diseños de políticas orientadas a impulsarlas y auxiliarlas, con el fin de incrementar su competitividad en un mundo globalizado y crezcan superando las condiciones de su tamaño (Parra et al 2018).

La mayoría de las MIPyMES que conforman el 99\% del tejido industrial, generan gran parte de los empleos, su productividad y desempeño es muy baja en comparación con las grandes empresas, ante esta situación se debe realizar el desarrollo de cadenas de producción que involucren compañías de diferentes tamaños, creando énfasis especial en las MIPyMES, es una exigencia obligatoria para la generación de empleos y salarios que reduzcan la diversidad de la economía de la región.

Las Pymes en México prevalecen en un mercado competitivo y con acceso restringido para adquirir tecnologías y programas administrativos vanguardistas y dificultad para 
obtener créditos, por lo que se puede adjudicar la responsabilidad solo al gobierno de promoverlas. Para lograrlo es necesario elaborar de forma conjunta un aparato productivo integral para mantener empresas sostenibles a largo plazo que creen y conserven los empleos y aumentando el capital para su desarrollo (Cruz, M. et al 2016).

La gran parte de los microempresarios no tienen desarrollado un plan de negocios y conocen su problemática estratégica, empresarial, pero ante limitaciones propias no las llegan a plasmar con claridad, lo que impide la indagación de alternativas integrales que solucionen sus problemáticas dentro de su organización (González 2007). En la actualidad las MIPyMES componen uno de los sectores con mayor productividad y muy representativo para los países procedentes o en vías de progreso debido a su aportación a la economía y a la creación de riquezas (Villegas \& Toro, 2010).

Durán (2017), dice que existen cinco temas relevantes que están relacionados con la alta tasa de fracasos de las Pymes: a) La mayoría de los dueños de estas empresas emprenden una actividad empresarial sin capacitación administrativa, b) Conocimiento contable y financiero, c) Manejo de inventarios, d) Ventas y atención al cliente y e) Manejo de recurso humano. El efecto es que después de cierto tiempo se dan cuenta de que no están teniendo las utilidades que inicialmente pensaron y la no recuperación de su capital inicial. Algunos crecen de manera exponencial en virtud de que la primera etapa es exitosa. Pero crecer implica no solamente en ampliación de la capacidad productiva sino también en administración y gestión empresarial.

Según la Ley para el desarrollo de la competitividad de la micro, pequeña y mediana empresa (2017), en la clasificación de las Pymes se incluyen productores agrícolas, ganaderos, forestales, pescadores, acuicultores, mineros, artesanos y de bienes culturales, así como prestadores de servicios turísticos y culturales. La clasificación de las Pymes es la siguiente:

Cuadro 1. Micro, pequeñas y medianas empresas, legalmente constituidas, con base en la estratificación establecida por la Secretaría, de común acuerdo con la Secretaría de Hacienda y Crédito Público y publicada en el Diario Oficial de la Federación.

\begin{tabular}{|c|c|c|c|}
\hline \multicolumn{5}{|c|}{ Estratificación por número de trabajadores } \\
\hline Sector/Tamaño & Industria & Comercio & Servicios \\
\hline Micro & $0-10$ & $0-10$ & $0-10$ \\
\hline Pequeña & $11-50$ & $11-30$ & $11-50$ \\
\hline Mediana & $51-250$ & $31-100$ & $51-100$ \\
\hline
\end{tabular}

Fuente: Tomado de la Ley para el Desarrollo de la Competitividad de la Micro, Pequeña y Mediana Empresa Cámara de Diputados del H. Congreso de la Unión (2017).

Las empresas MIPyMES en el área de agroindustrias, representan en el Estado de Chihuahua el fragmento de la economía que aporta la mayor cifra de unidades económicas y personal empleado; de ahí la relevancia que reviste este tipo de empresas y la necesidad de fortificar su desempeño en cuanto a capacidades productoras, emprendedoras $\mathrm{y}$ comerciales. 
Da Silva et al (2013) hace referencia que el sector agroindustrial se define como el subconjunto del sector manufacturero que procesa materias primas y productos intermedios agrícolas, forestales y pesqueros. De este modo, el sector agroindustrial incluye fabricantes de alimentos, bebidas y tabaco, textiles y prendas de vestir, muebles y productos de madera, papel, productos de papel e impresión, además de plástico y productos de plástico. A su vez, la agroindustria forma parte del concepto más amplio de agronegocio, que incluye proveedores de insumos para los sectores agrícola, pesquero y forestal, además de distribuidores de alimentos y de productos no alimentarios procedentes de la agroindustria. No se conoce en forma general y específica, las capacidades productoras, emprendedoras y comerciales, de las MIPyMES del sector Agroindustrial del municipio de Delicias, Chihuahua, se carece de un análisis empresarial de sus capacidades y necesidades que los conduzca a su desarrollo sostenible.

Es importante que la Facultad de Ciencias Agrícolas y Forestales contribuya al desarrollo económico del municipio de Delicias, Chihuahua y su situación actual. Sin embargo, no tiene información actualizada. Para lo cual este estudio de caso va a contribuir a darle solución al problema de investigación que contiene las capacidades productoras, emprendedoras y comerciales de las MIPyMES del sector agroindustrial de Delicias, Chihuahua.

De esta forma se pretende obtener información para analizar las condiciones actuales de las agroindustrias del municipio de Delicias y aprovechar las oportunidades empresariales, a través de sus fortalezas, oportunidades de innovación tecnológica, procesos y tener una base de datos de las MIPyMES del sector agroindustrial para conocer sus capacidades y necesidades, para proponer estrategias de desarrollo. El objetivo general de esta investigación es contribuir al desarrollo económico de la región a través de identificar capacidades productivas y empresariales de las MIPyMES en el municipio de Delicias, Chihuahua.

\section{MATERIALES Y MÉTODOS}

El área de trabajo está localizada en las MIPyMES del sector agroindustrial, en el municipio de Delicias, Chihuahua. El tipo de investigación fue descriptiva y correlacional. Las variables que se evaluaron fueron capital de recursos humanos, capital de recursos productivos y activos no tangibles, capital financiero, capital contable, capital de comercialización y capital relacional. Las variables que se evaluaron fueron capital de recursos humanos, capital de recursos productivos y activos no tangibles, capital financiero, capital contable, capital de comercialización y capital relacional.

Se llevó acabo de la siguiente manera:

1. Se aplicaron cuestionarios directamente a los empresarios de las MIPyMES del sector Agroindustrial. Utilizando un enfoque cuantitativo y cualitativo.

2. El tipo de investigación que se utilizo es descriptiva y correlacional. (Hernández et al 2007).

3. Con los resultados obtenidos se realizó una base de datos actualizada de MIPyMES agroindustriales.

4. Se utilizó el programa de SPSS IBM v22 para el análisis estadístico de las variables. 
5. Se realizó un análisis de fiabilidad de alfa de Cronbach, utilizando las escalas de Likert (George \& Mallery, 2003).

\section{RESULTADOS}

La presente investigación tiene por objetivo identificar, analizar y describir MIPyMES agroindustriales de Delicias, Chihuahua, registradas en las organizaciones empresariales acreditadas y las no registradas. Cuantificar las capacidades considerando el capital humano productivo y administrativo, capital financiero, contable, de comercialización y relacional, así como cuantificar las necesidades empresariales; producción, capacitación, créditos, comercialización y manejo de residuos.

Por otra parte, se presentarán resultados obtenidos del proyecto MIPyMES agroindustriales a cada empresario del municipio de Delicias, Chihuahua. Los principales resultados del estudio se realizaron con una base de datos con el programa Statistical Program for Social Science (SPSS IBM V23), fue con base a la captura de la información cualitativa y cuantitativa de 33 preguntas del cuestionario que se aplicó a 25 agroindustrias, para el procesamiento de los datos. La técnica de muestreo utilizada fue la bola de nieve.

Se codifico el cuestionario resultando 62 variables cuantitativas y 126 variables cualitativas. La técnica de aplicación del cuestionario fue una entrevista personal a 20, telefónica 2 y por e-mail 3. Se verifico que las variables cualitativas fueran capturadas como tal, así como las escalares. Así mismo se verifico que ninguna variable tuvo valores perdidos. Se identifica las capacidades productivas y necesidades empresariales actuales en las MIPyMES del sector agroindustriales del municipio de Delicias, Chihuahua.

Los principales resultados son los siguientes:

Gráfica 1. Clasificación de MIPyMES por número de trabajadores.

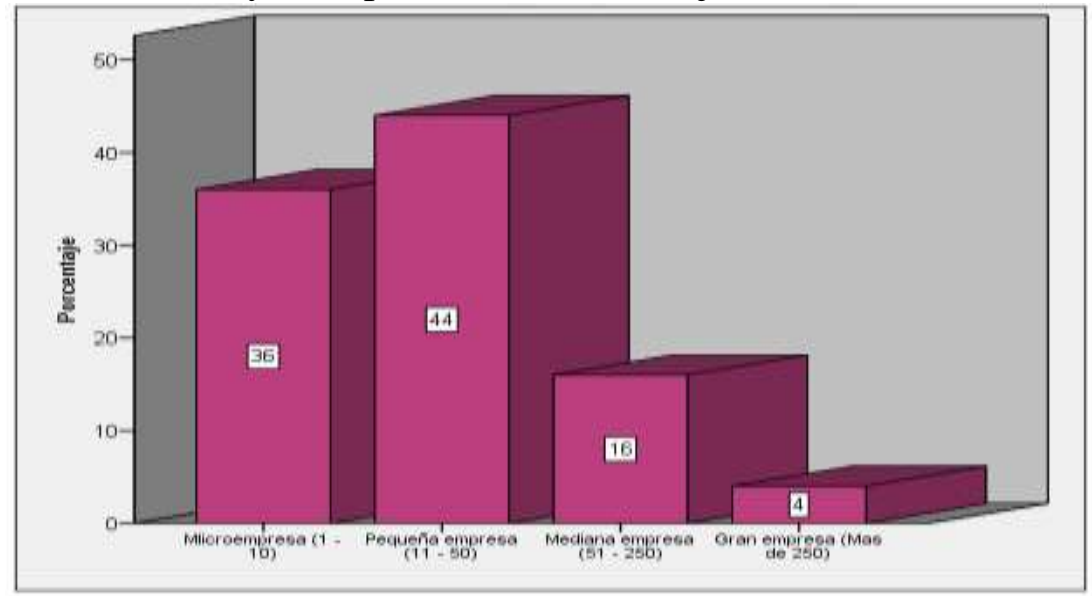

Fuente: Elaboración propia

La estructura de las empresas que fueron objeto y muestra de la investigación fueron $36 \%$ microempresas, $44 \%$ pequeñas empresas, $16 \%$ de medianas empresas y una gran empresa que representa el 4\%. Esta distribución es representativa en la industria local respecto a la participación relativa de cada tipo de empresa (gráfica 1). 
Cuadro 2. ¿Cuáles son los departamentos que tiene la empresa?

\begin{tabular}{|c|c|c|c|c|}
\hline Departamento & $\begin{array}{c}\text { Número de } \\
\text { trabajadores }\end{array}$ & $\begin{array}{c}\text { Participación } \\
\text { relativa }\end{array}$ & Media & Rango \\
\hline Producción & 709 & $65.71 \%$ & 28.36 & 350 \\
\hline Administración & 162 & $15.01 \%$ & 6.48 & 99 \\
\hline $\begin{array}{c}\text { Recursos } \\
\text { Humanos }\end{array}$ & 13 & $1.20 \%$ & .52 & 3 \\
\hline Ventas & 94 & $8.71 \%$ & 3.76 & 40 \\
\hline $\begin{array}{c}\text { Mercadotecnia } \\
\text { Seguridad del } \\
\text { Trabajo y PS }\end{array}$ & 3 & $0.74 \%$ & .32 & 1 \\
\hline Finanzas & 24 & $0.28 \%$ & 12 & 7 \\
\hline \begin{tabular}{c} 
Contabilidad \\
\hline $\begin{array}{c}\text { Total de } \\
\text { trabajadores }\end{array}$
\end{tabular} & 29 & $2.22 \%$ & .96 & 5 \\
\hline
\end{tabular}

Fuente: Elaboración propia con datos obtenidos en campo.

El total de trabajadores de la muestra fue de 1079 en donde el $65.71 \%$ estaban en el departamento de producción y el $15.01 \%$ en administración (cuadro 2).

Gráfica 2. Clasificacion de MIPyMES por giro.

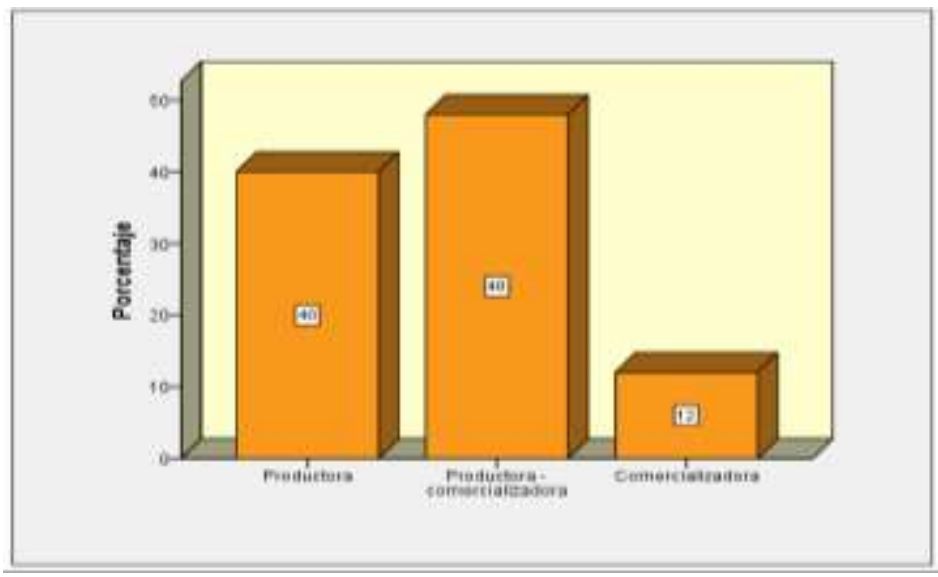

Fuente: Elaboración propia. 
La clasificación de las empresas por giro, que fueron objeto de estudio y muestra de la investigación, se distribuyen en un $40 \%$ productoras, $48 \%$ productora comercializadora y $12 \%$ comercializadora.
Cabe destacar que la clasificación de productora - comercializadora es más grande porque existe una empresa que representa el $33 \%$ de actividad productiva (gráfica 2).

Gráfica 3. ¿Cuenta con permiso de suelo?

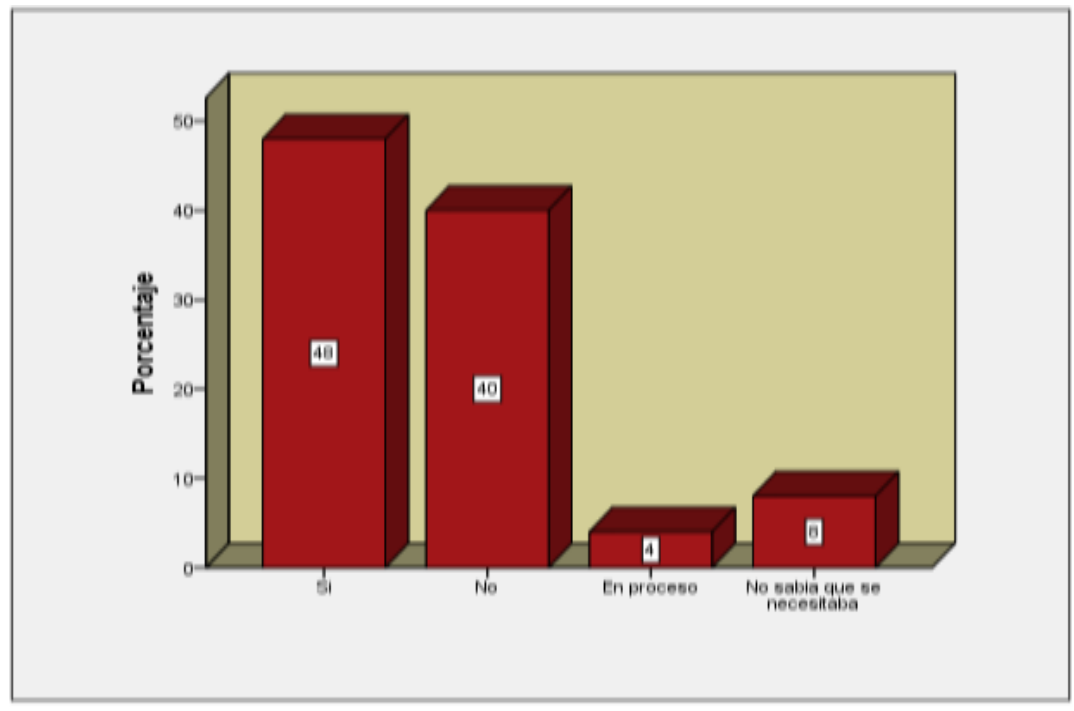

Fuente: Elaboración propia.

Un elemento clave de normatividad es el permiso de uso industrial del suelo. Las empresas manifestaron que de las 25 empresas entrevistadas, el $48 \%$ dijo que cuenta con permiso de suelo, el $40 \%$ no lo tiene, un $4 \%$ está en proceso y el $8 \%$ comenta que no sabían que se necesitaba (gráfica 3). 


\section{Gráfica 4. ¿Cuenta con un plan estratégico establecido?}

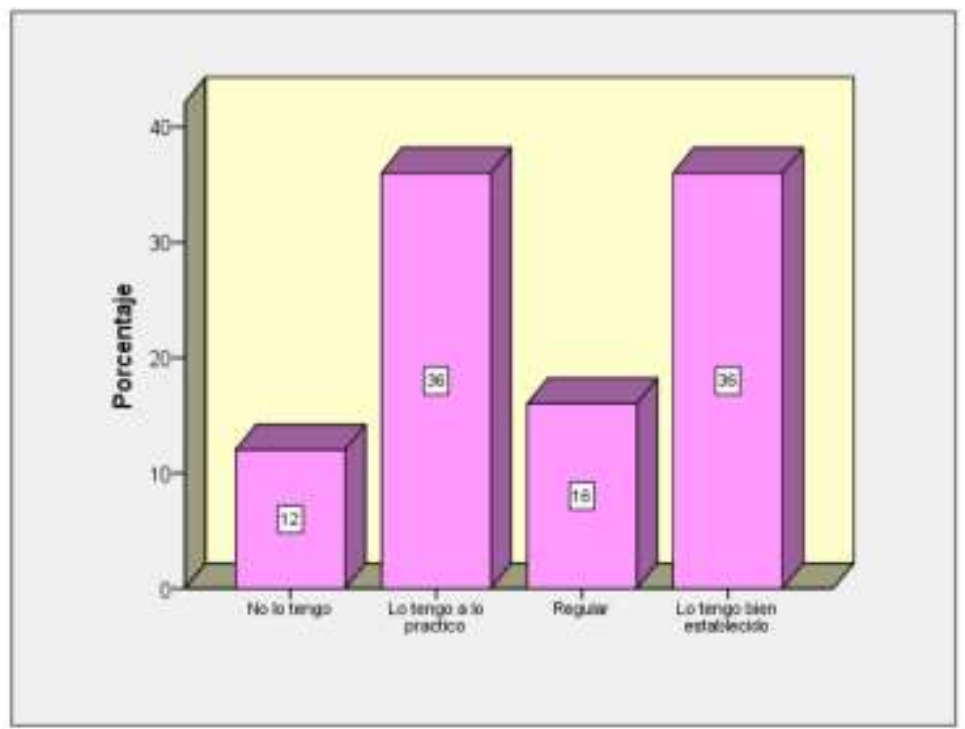

Fuente: Elaboración propia

Un proceso sistemático y dinámico clave que formula la dirección estratégica basada en la competitividad, continuidad en el mercado y en el desarrollo sustentable, es la planeación estratégica. Las empresas manifestaron que, de las 25 empresas entrevistadas, el $36 \%$ dijo que cuenta con un plan estratégico bien establecido, el $16 \%$ lo tiene regular, el $36 \%$ comenta que lo tiene a lo práctico y el $12 \%$ expone que lo tiene. El $52 \%$ no tienen una planeación estratégica como tal y tienen objetivos y metas de producción y ventas de acuerdo a sus compromisos y no como un documento de guía estratégica que implica la producción. En tanto el $12 \%$ que pertenecen a las microempresas, manifestaron que no tiene nada de planeación estratégica (gráfica 4).

Para verificar la asociación entre misión y la planeación estratégica se condujo una prueba de hipótesis utilizando la chi cuadrada para medir el grado de asociación entre la variable la planeación estratégica y la misión.

Ho: La planeación estratégica y la misión no están asociadas.

Ha: La planeación estratégica y la misión si están asociadas.

Al realizar la prueba se tuvo:

Cuadro 3. Pruebas de chi-cuadrado.

\begin{tabular}{|c|c|c|c|}
\hline Prueba & Valor & Gl & $\begin{array}{c}\text { Sig. asintótica } \\
\text { (bilateral) }\end{array}$ \\
\hline Chi-cuadrado de Pearson & $18.333^{\mathrm{a}}$ & 9 & .031 \\
\hline Razón de verosimilitudes & 20.203 & 9 & .017 \\
\hline Asociación lineal por lineal & 11.542 & 1 & .001 \\
\hline N de casos válidos & 25 & & \\
\hline
\end{tabular}

Fuente: elaboración propia con datos obtenidos en campo. 
El valor de chi cuadrada calculada $=18.33$

El valor de chi cuadrada de tablas con $\mathrm{gl}=9$, alfa $=0.05$ es igual a 16.919

Por lo tanto, existe suficiente evidencia estadística para rechazar la hipótesis nula y trabajar con la hipótesis alterna. A continuación, se analiza la tabla cruzada de las variables planeación estratégica y misión.

Cuadro 4. Capacitación urgente de acuerdo a la clasificación de MIPyMES por giro.

\begin{tabular}{|c|c|c|c|c|}
\hline & \multicolumn{3}{|c|}{ Clasificación de MIPyMES por giro. } & Productora - \\
\hline $\begin{array}{c}\text { Área de } \\
\text { capacitación }\end{array}$ & Productora & $\begin{array}{c}\text { Promercializadora } \\
\text { Comercializadora }\end{array}$ & Total \\
\hline Producción & 10 & 12 & 2 & 24 \\
\hline Administración & 7 & 10 & 3 & 20 \\
\hline $\begin{array}{c}\text { Recursos } \\
\text { Humanos }\end{array}$ & 4 & 9 & 1 & 14 \\
\hline Ventas & 7 & 10 & 2 & 19 \\
\hline Mercadotecnia & 2 & 6 & 2 & 10 \\
\hline $\begin{array}{c}\text { Seguridad del } \\
\text { trabajo y PS }\end{array}$ & 2 & 7 & 2 & 11 \\
\hline Finanzas & 4 & 10 & 3 & 22 \\
\hline Contabilidad & 9 & 10 & 3 & 17 \\
\hline
\end{tabular}

Fuente: elaboración propia con datos obtenidos en campo.

De las 35 empresas, indicaron que existe una necesidad urgente de capacitación. 24 de las cuales manifestaron que requieren urgentemente capacitación en el área de producción. En tanto que 22 empresas dijeron que es necesariamente en contabilidad, 20 de ellas revelaron que urge la capacitación en el área de administración, y 19 empresas consideran necesariamente la capacitación en el área de ventas (cuadro 4).

\section{CONCLUSIÓN}

El alcance de la base de datos obtenida de las empresas encuestadas fueron microempresas y pequeñas empresas. En tanto a la distribución de los trabajadores en la planta fue en el departamento de producción. Se observa un porcentaje muy elevado de trabajadores destinados a labores administrativas lo que ocasiona un incremento en los costos de mano de obra. Así mismo, se observó que más del $50 \%$ no cumplen con la normatividad del permiso de uso de suelo industrial, esto hace necesario cambiar el modelo de gestión empresarial.

Al analizar el nivel de estudios de la muestra se observó que la participación de los trabajadores en el departamento de producción es similar al porcentaje de los que no tienen el nivel de licenciatura. Las empresas demandaron urgentemente capacitación en orden prioritario en producción, contabilidad, administración y ventas. De acuerdo a la clasificación de las empresas por giro, la mayoría de ellas son 
productoras y comercializadoras de productos que son de origen agrícola y pecuario.

El estudio alcanzo los objetivos declarados al conocer las capacidades productivas y empresariales de las MIPyMES del municipio de Delicias, Chihuahua. Entre sus principales fortalezas se encontró que las empresas son dirigidas por personas con capacidad emprendedora y potencial para transformar de mejor forma, estrategias que les permita alcanzar un futuro deseable para la organización. Esto, hace que utilicen sus recursos de una forma adecuada para alcanzar sus objetivos, tales como recurso humano, equipo e infraestructura, ventas y canales de distribución. Sin embargo, concluimos que, se tiene que trabajar más en sus debilidades, capacitación en diferentes áreas, planeación estratégica e integración a las diferentes cámaras y organizaciones empresariales.

\section{LITERATURA CITADA}

Arana, D. (2018). Pymes mexicanas, un panorama para 2018. International Finance Corporation. https://www.forbes.com.mx/author/inter national-fin/. 15 de agosto del 2019. 1:50 pm. México Forbes.

Cruz, M. et al (2016). ¿Por qué no crecen las Micro y Pequeñas empresas en México? Universidad Tecnológica de Tecámac, San Juan del Río Querétaro, 2016.

Da Silva C., A., Doyle B., Shepherd, A., W., Jenane C., \& Miranda da C., S., Agroindustrias para el desarrollo, Organización de las Naciones Unidas para la alimentación y la agricultura Roma, (2013).
Durán Mena (2017). ¿Por qué naufragan los pequeños negocios? Revista Forbes, México

https://www.forbes.com.mx/author/cdur anm/. 16 de agosto de 2019. 8:00 pm

George, D., \& Mallery, P. (2003). SPSS for Windows step by step: A simple gude and reference, 11.0 update. Obtenido de wps.

Ablongman.com/wps/media/objects/385 . Georige 4answers pdf.

González Alvarado, R. (2007). Análisis estratégico de la empresa Carne Seca el "Abigeo" ubicada en el municipio de Delicias, Chihuahua. (Tesis inédita de maestría). Universidad Autónoma de Chihuahua, Facultad de Ciencias Agrícolas y Forestales, Delicias, Chihuahua.

Hernández Sampieri, Roberto Fernández Collado Carlos; Baptista Lucio, Pilar. Metodología de la Investigación. Mc Graw Hill, México 1997.

INEGI (2018). Contribución de las Pymes a la economía mexicana. Aguascalientes, Aguascalientes.

Ley para el Desarrollo de la Competitividad de la Micro, Pequeña y Mediana Empresa Cámara de Diputados de H. Congreso de Unión Secretaria General Secretaria de Servicios Parlamentarios Ultima Reforma DOF 19-05-2017.

Parra, T., D., J., A., Loera, E., M., \& Hinojosa, G., C. (2018). Factores que promuevan la competitividad de las Mipymes en la región de cd. 
Cuauhtémoc, Chihuahua, México, Red

Internacional de Investigadores en Competitividad, 5(1).

Recuperado en: https://www.rii co.net/index.php/riico/article/view/690
Villegas, D. I. \& Toro, I. D. (2010). Las Pymes: una mirada a partir de la experiencia académica del MBA. Revista MBA EAFIT, pp. 86-101. Disponible en: http://www.eafit.edu.co/revistas/revistam ba/Documents/pymes-mirada-a-partirexperiencia-academica-mba.pdf4

Copyright (c) 2020 Magaña Magaña José Eduardo, Chacón Márquez Aracely, Villarreal Ramirez Victor Hugo, Herm osillo Nieto José Javier, Ortega Montes Fabiola Iveth

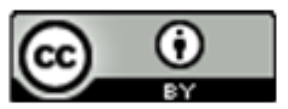

Este texto está protegido por una licencia licencia CreativeCommons 4.0.

Usted es libre para Compartir — copiar y redistribuir el material en cualquier medio o formato- y Adaptar el documento — remezclar, transformar y crear a partir del material- para cualquier propósito, incluso para fines com erciales, siempre que cumpla la condición de:

Atribución: Usted debe dar crédito a la obra original de manera adecuada, proporcionar un enlace a la licencia, e indicar si se han realizado cambios. Puede hacerlo en cualquier forma razonable, pero no de forma tal que sugiera que tiene el apoyo del licenciante o lo recibe por el uso que hace de la obra.

Resumendelicencia - Textocompletodelalicencia 